\title{
ARTÍCULO DE REVISÓN
}

\section{Análisis de las medidas educativas dispuestas en Paraguay en tiempos de pandemia desde la perspectiva de los derechos de la niñez y la adolescencia}

\author{
Analysis of the educational measures arranged by Paraguay during the \\ COVID-19 pandemic from childhood and adolescence right's perspective
}

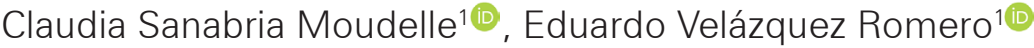

\begin{abstract}
Resumen
La pandemia del COVID-19 tuvo impacto social y económico a escala global. El sector educativo no estuvo exento y el modelo educativo tradicional sufrió cambios intempestivos sin precedentes. Se analizaron las tensiones surgidas entre las políticas educativas y la efectividad del derecho a la educación de niñas, niños y adolescentes en tiempos de pandemia. Se estudiaron dos actos normativos emanados de la autoridad educativa nacional: el Plan Educativo Nacional en tiempos de Pandemia y la Resolución N602/2020, desde una lectura basada en los principios y preceptos normativos nacionales e internacionales que garantizan el derecho a la educación, especialmente la Constitución Nacional; Ley N¹264/98 General de Educación; Ley N57/90, Convención de las Naciones Unidas sobre los Derechos del Niño; y Ley N 1680/2001, Código de la Niñez y la Adolescencia. Se tuvieron en cuenta: las observaciones del Comité de los Derechos del Niño; las recomendaciones del Fondo de Naciones Unidas para la Infancia (UNICEF) y de la Organización de las Naciones Unidas para la Educación, Ciencia y Cultura (UNESCO). A través de la revisión documental y análisis jurídico se encontró que: el plan es teórico y no contempló estrategias universales; la llegada a las familias fue inexistente; la propuesta no es flexible e individualizada; la evaluación se enfocó en el rendimiento y no el desarrollo de la personalidad; se observaron limitaciones de acceso a internet y formación docente; no se consideró la autonomía y participación de los niños. Se hicieron recomendaciones en base a los hallazgos.
\end{abstract}

1 Universidad Iberoamericana, Paraguay.

Correspondencia: Eduardo Velázquez Romero. Email: eduvelrom@gmail.com

Recibido: 30/09/2020. Aceptado: 20/10/2020.

DOI: http://dx.doi.org/10.26885/rcei.9.1.189

Artículo publicado en acceso abierto bajo Licencia Creative Commons. 
Palabras clave: niñas, niños y adolescentes, derecho a la educación, pandemia, políticas educativas.

\section{Abstract}

The global pandemic of COVID-19 has had social and economic impact in a global scale. The educational sector has not been the exception and the traditional models of education went through an unprecedented process of changes. In this context, the following article analyze the key aspects in the middle of a stressed situation between the educational policies and the effectiveness of the right of children and adolescents to education in times of pandemic. The start point of this study are the two normative acts that come from the national authority in educational matters: the National Educational Plan for pandemic times and the Resolution $N^{\circ} 602 / 2020$. From a reading based on the principles and normative precepts at national and international level that guarantee the right to education, specially the National Constitution; the General Law of Education $N^{\circ}$ 1264/98; Law N $N^{\circ} 1680 / 2001$ that approves the United Nations Convention on the rights of the Child (from now on UNCC); and the Law $\mathrm{N}^{\circ}$ $1680 / 2001$ that approves the Code of Children and Adolescent (from now on (CA). At the same time, there will be a showing of the plan considering the comments of international organizations in the subject such as United Nations International Children's Emergency Fund (UNICEF) and the United Nations Educational, Scientific and Cultural Organization (UNESCO). It is proposed that through a documental review and analysis of law, to be able to offer comments and proposals with a right approach that help to reduce as much as possible the appearance of vulnerabilities to the rights of children and adolescents.

Keywords: girls, boys and adolescents, right to education, pandemic, educational policies.

\section{INTRODUCCIÓN}

El 11 de marzo de 2020 la Organización Mundial de la Salud (OMS) declara la pandemia por el brote de enfermedad del Coronavirus (COVID-19) a nivel internacional. Los gobiernos en su mayoría toman medidas de prevención para evitar la propagación del virus y el consecuente aumento de contagios y fallecidos. Una de las principales medidas asumidas fue la del aislamiento físico preventivo con todo lo que ello implicaba: la suspensión de cualquier tipo de actividades que conlleven aglomeración de personas: eventos multitudinarios, fiestas, reuniones, clases presenciales, entre otros.

En ese contexto, la suspensión de las clases presenciales para niñas, niños y adolescentes fue una de las tantas medidas asumidas. A nivel nacional, el 11 de marzo de 2020, el Ministerio de Educación y Ciencias, órgano rector del 
Sanabria Moudelle, C., \& Velázquez Romero, E. Medidas educativas dispuestas en Paraguay en tiempos de pandemia desde la perspectiva de los derechos de la niñez y la adolescencia

sistema educativo, dispuso por Resolución N³08/2020 la suspensión de clases presenciales en todas las instituciones educativas tanto de gestión pública como privada.

Pues bien, a partir de esta medida por la cual se suspenden las clases presenciales, conviene entrar a analizar de manera pormenorizada, de qué forma se vio afectado el derecho a la educación o más específicamente, su efectividad.

Para hablar de la efectividad del derecho a la educación en tiempos de pandemia, y como punto de partida nos preguntamos: ¿Puede la escuela ir a casa? Si es así, ¿cuáles serían las condiciones? ¿Cómo ayudar a las familias para adaptarse a este nuevo cambio, a convivir con la escuela? ¿Cómo proporcionar a las familias, condiciones para apoyar el proceso educativo? ¿Se contempla el acompañamiento presencial a las familias desde el inicio de la crisis sanitaria, entendiendo que en contextos vulnerables, es más efectiva la llegada presencial?

En línea con la efectividad, existen otras preguntas iniciales que orientan el análisis de toda política pública: ¿Cuál es la mirada hacia los potenciales beneficiarios de las acciones en las políticas educativas? ¿Se tiene en cuenta a la infancia o a las infancias; la adolescencia o las adolescencias? ¿Se piensa en diversidad o en un grupo homogéneo?

Siguiendo en la lógica de comprobar la efectividad del derecho, parece necesario revisar cuál es el interés superior del niño hablando de su derecho a la educación, y de qué manera este interés ha sido materializado en el plan. Es igualmente importante, analizar si se establecieron las clases por niveles teniendo en cuenta las recomendaciones de salud para el desarrollo evolutivo de las niñas, niños y adolescentes.

La presente revisión se centra principalmente en dos actos normativos del Ministerio de Educación y Ciencias, siendo estos: el "Plan de Educación en tiempos de pandemia" -en adelante, el Plan- y en la Resolución N 602/2020 "Por la cual se aprueba el documento Orientaciones para evaluar el aprendizaje de los estudiantes en los diferentes niveles y modalidades del Sistema Educativo Nacional en tiempos de pandemia".

Como parte del estudio se consideran algunos aspectos que son relevantes a la hora de corroborar la garantía del Derecho a la Educación en tiempos de Pandemia: i. La integralidad de la propuesta educativa, que atienda a las necesidades del cien por ciento de la población de niñas, niños y adolescentes en edad escolar (currículo basado en competencias mínimas para el año, condiciones socioeconómicas, etapa evolutiva de la niña, niño, adolescente); ii. El rol de las familias y las estrategias de empoderamiento y fortalecimiento teniendo en cuenta que el éxito de la política educativa descansa en ella; iii. El sistema de evaluación que se propone y iv. La participación de los propios niños, niñas y adolescentes en la propuesta educativa. 
El estudio se hace desde la mirada del compromiso de Paraguay como Estado Parte de la Convención de las Naciones Unidas sobre los Derechos del Niño (en adelante CDN), con lo cual, la perspectiva de estudio y los planteamientos se realizan en este trabajo tomando como plataforma de base los principios y preceptos del citado instrumento jurídico. Se realiza así una revisión documental de los actos normativos supra mencionados enfocando el estudio de este artículo en encontrar los puntos en común y las tensiones que se generan entre las normas adoptadas por la Autoridad Nacional en materia de educación durante la Pandemia y el marco Normativo Nacional e Internacional en materia de acceso a la educación para así proponer finalmente sugerencias e identificar oportunidades de mejora

\section{ReVISIÓN DOCUMENTAL}

La revisión documental parte de un análisis del contexto en el cual se diseña el plan y en el cual se publica, de tal forma a lograr una mejor comprensión de las variables que se presentan en el documento base de análisis de este artículo y las propuestas de reflexión desde la perspectiva de derecho. Se presenta por tanto el contexto en cuanto a medidas gubernamentales asumidas desde la instancia educativa estatal

\section{Las Medidas adoptadas en el Sistema educativo paraguayo duRANTE la PANDEMIa del COVID-19}

Declarada la situación de emergencia a nivel nacional por Decreto del Poder Ejecutivo N 3456 "Por el cual se declara Estado de Emergencia Sanitaria en todo el territorio nacional para el control del cumplimiento de las medidas sanitarias dispuestas en la implementación de las acciones preventivas ante el riesgo de expansión del Coronavirus (COVID-19)", de fecha 16 de marzo de 2020, y la consecuente suspensión de clases presenciales en todas las instituciones educativas de gestión pública y privada en todo el territorio nacional, se inicia un proceso de transición con el fin de pasar de la modalidad tradicional de clases presenciales a las clases virtuales. Es preciso puntualizar que, las clases en modalidad totalmente virtual para la educación escolar básica y nivel medio, dirigida a niñas, niños y adolescentes, no tienen precedente alguno a nivel mundial, vale decir, al proponer una respuesta en este sentido las autoridades se vieron desprovistas de experiencias, precedentes, modelos o estudios científicos que orienten las medidas a ser implementadas.

Es preciso puntualizar que, las clases en modalidad virtual para la educación escolar básica y nivel medio, dirigida a niñas, niños y adolescentes, no tienen precedente alguno a nivel mundial, vale decir, al proponer esta opción como respuesta las autoridades se vieron desprovistas de experiencias, modelos o estudios científicos que orienten las medidas a ser implementadas.

Sumado a lo expuesto debe tenerse presente el propio contexto 
educativo de Paraguay, que, como parte de América Latina, se caracteriza por problemáticas propias en su sistema educativo tales como:

Las grandes brechas en los resultados educativos, que se relacionan con una desigual distribución de los docentes, en general, y de los docentes mejor calificados, en particular, en desmedro de países y regiones con menores ingresos y de zonas rurales, las que suelen concentrar además a población indígena y migrante (UNESCO, 2016a; Messina \& García, 2020 citado en CEPALUNESCO, 2020).

Muchos de los problemas que afectan a la región en el ámbito educativo, ya citados precedentemente, se acentuaron con la pandemia del COVID-19. A esto se suma el proceso propio que vivió el sistema educativo nacional y que atravesó por una transición que en forma general puede resumirse en tres fases:

a. Fase Inicial-Marzo 2020: a nivel nacional el inicio de la cuarentena se caracterizó por la situación de incertidumbre que impacto fuertemente en la sociedad. El sistema educativo vivió una interrupción intempestiva en su cronograma de clases el cual estaba en etapa de inicio tanto en el sector privado como en el sector oficial.

b. Fase Intermedia- Abril 2020: se reinician las clases en forma gradual (de manera virtual) sobre todo en las instituciones educativas del sector privado, alargándose aún más el retorno a clases en el sector público. Como nota característica de esta fase se resaltan:

b.1. Aumento de la tensión por parte de los padres de familia por un descontento con la modalidad virtual de enseñanza en instituciones educativas públicas y privadas y el nuevo rol que los tocaba asumir incluyendo un acompañamiento mucho más cercano al proceso educativo de sus hijos e hijas;

b.2. Aumento de la tensión entre padres y directivos de instituciones del sector privado con respecto al cumplimiento de pago de cuotas y compromisos administrativos, tema que tuvo notable repercusión nacional y tuvo asidero de discusión incluso a nivel de Congreso Nacional pues se presentaron proyectos de leyes que abogaban por la exoneración de pago de aranceles en las instituciones educativas privadas. Así también se puede citar como antecedente la intervención del poder judicial con respecto a decidir si correspondía o no el pago de cuotas, ambas instancias no tuvieron curso favorable y se instó a los sectores en conflicto a recurrir a la negociación de partes como herramienta de solución de sus diferencias;

b.3. Empieza a resentirse la falta de acceso y falta de conectividad a internet en muchas localidades del país lo cual 
dificultaba el desarrollo de clases virtuales.

b.4. Reclamo por parte del sector estudiantil y del sector docente demandando ser consultados para la toma de decisiones y mayor participación.

c. Implementación de la Modalidad Virtual y Puesta en Marcha del Plan Educativo - Junio 2020: ya habiendo transcurrido tres meses y vivenciadas las "tensiones" mencionadas el MEC publica el Plan y oficialmente se inicia su ejecución.

A partir de esta última fase y ya con un documento oficial publicado por el MEC puede decirse que culmina el periodo de transición de lo presencial a lo virtual y efectivamente se inicia un nuevo modelo educativo totalmente virtual. Habiéndose presentado el contexto, se presenta el estudio del Plan desde la perspectiva de protección de los derechos de la niñez y adolescencia.

\title{
Análisis del Plan Educativo. Sus objetivos, estrategias y Viabilidad de ejecución
}

Revisando los objetivos plasmados en el Plan (MEC, 2020), nos encontramos con un objetivo general y siete objetivos específicos. Es correcta la lógica que propone el plan en cuanto a cómo plantea su objetivo general y los consecuentes específicos. El objetivo general apunta a:

\begin{abstract}
Garantizar el derecho a la educación mediante la prestación del servicio educativo a los estudiantes matriculados en el sistema educativo nacional durante el periodo de emergencia sanitaria, a través de la modalidad de educación a distancia que facilite el proceso de enseñanza - aprendizaje. (MEC, 2020, p. 11)
\end{abstract}

Este objetivo refuerza la idea de que el plan fue expedido con el fin principal de garantizar que el servicio educativo pueda prestarse sin interrupciones, reconociendo que la base de poder ejercer la educación como derecho humano parte de su acceso mismo y que a partir de allí subyacen otros derechos. Pasando a los objetivos específicos, nos encontramos con una serie de propuestas que cubren todas y cada una de las etapas del proceso educativo desde una mirada general y universal de la educación. Hasta aquí no se encuentran mayores dificultades, ahora bien ahondando más en la lectura del plan y siguiendo con a revisión es en el Apartado VI - sobre las fases y estrategias de implementación donde empiezan a denotarse las primeras fragilidades en cuanto a que no lograrían cumplirse con el gran porcentaje de escala de beneficiarios del 90\% (MEC, 2020) que el propio plan propone, con lo cual puede decirse que por sí mismos los objetivos resultan ser bastante desafiantes por el dificultoso escenario marcado por la pandemia por un lado, y por otro, por las condiciones de base considerando la escasa formación docente 
Sanabria Moudelle, C., \& Velázquez Romero, E. Medidas educativas dispuestas en Paraguay en tiempos de pandemia desde la perspectiva de los derechos de la niñez y la adolescencia

en el uso de las tecnologías y recursos; formación inespecífica en metodologías de enseñanza virtual; acceso a medios; en pocas palabras, lo que se pretende podría exceder a las posibilidades del sector educativo, sobre todo en el ámbito público.

Considerando así que el plan ya se encuentra en vigencia y en ejecución, se debe velar entonces para que el mismo se implemente teniendo como principios orientadores, aquellos enunciados en los Arts. 3, 5, 12 de la CDN: a) Priorizar el interés superior del niño; b) Considerar la autonomía progresiva, viendo al niño "realmente" como sujeto de derechos; c) El derecho a la participación, el derecho a ser oído, inclusive en el proceso de construcción de la respuesta.

Se requiere sin duda, una mirada a las características del Derecho a la Educación en la legislación especializada, su interpretación en base al desarrollo de observaciones y recomendaciones de los órganos de los tratados. Se debe partir para este análisis de lo que establece la Constitución Nacional, que en el Art. 73 "Del derecho a la educación y sus fines", dispone cuanto sigue: "Toda persona tiene derecho a la educación integral y permanente, que como sistema y proceso se realiza en el contexto de la cultura de la comunidad". Este primer apartado proporciona dos características fundamentales de la educación, por un lado la integralidad, y por el otro, la de ser permanente. En el primer caso, podemos entender que la integralidad apuntaría a mirar todas las dimensiones de la persona (física, emocional, cognitiva); y en el segundo, la de ser permanente nos daría la condición de que no puede faltar, es un servicio que debe ser proporcionado a la persona sin interrupciones, o quizás pese a cualquier contingencia o contexto.

Vinculadas a la norma constitucional, encontramos los principios que proporciona la CDN que deben ser aplicadas en su conjunto, teniendo en cuenta el Principio del Interés Superior del Niño (Art. 3); Principio de Autonomía Progresiva (Art. 5); Principio de Supervivencia y Desarrollo (Art. 6); Principio de la Participación (Art. 12); como así también tendrán el resguardo de garantizar que la educación del niño esté dirigida a:

a) desarrollar la personalidad, las aptitudes y la capacidad mental y física del niño hasta el máximo de sus posibilidades... d)preparar al niño para asumir una vida responsable en una sociedad libre, con espíritu de comprensión, paz, tolerancia, igualdad de los sexos y amistad entre todos los pueblos, grupos étnicos, nacionales y religiosos y personas de origen indígena"(Art. 29 CDNA).

Como bien reflexiona Romero de Velázquez (2011) en relación con la implicancia del "interés superior del niño" en nuestro marco normativo nacional y el sistema educativo:

Considero que al incorporar los principios del interés superior del niño y de la protección efectiva de sus derechos, las autoridades nacionales, judiciales, el MEC, las familias y la sociedad toda deben prevalecer el ejercicio pleno del 
derecho a recibir una educación de calidad que les corresponde legítimamente a los niños y adolescentes de nuestro país, promoviendo políticas públicas que garanticen el cumplimiento real y, en el caso de existir conflictos entre derechos o intereses, obrar siempre a favor de los derechos de los niños, respetando el carácter prevaleciente de sus derechos. (p. 193)

En línea con lo apuntado, cabe mencionar la interpretación orientadora proporcionada por el Comité de los Derechos del Niño en la Observación General $N^{\circ} 14$, sobre el derecho del niño a que su interés superior sea una consideración primordial:

33. El interés superior del niño se aplicará a todos los asuntos relacionados con el niño o los niños y se tendrá en cuenta para resolver cualquier posible conflicto entre los derechos consagrados en la Convención o en otros tratados de derechos humanos. Debe prestarse atención a la búsqueda de posibles soluciones que atiendan al interés superior del niño. Ello implica que los Estados tienen la obligación de aclarar, cuando se adopten medidas de aplicación, cuál es el interés superior de todos los niños, incluidos los que se encuentren en situación de vulnerabilidad. (CRC/C/GC/14, 29 de mayo de 2013, párrafo 33, p. 265)

El mismo Comité de los Derechos del Niño (Abril, 2020), en sus recomendaciones emitidas a los Estados a raíz de la Pandemia pone en evidencia dos elementos que consideramos elementales a tener en cuenta en toda medida que afecte o restrinja derechos y que serían una medida del cumplimiento del Principio del Interés Superior: la proporcionalidad y la temporalidad. Por un lado, entonces se requiere que toda medida sea estrictamente necesaria para velar en equilibrio por la vigencia de todos los derechos de la persona en su conjunto, por ejemplo, en este caso, se aplica una medida restrictiva respecto a la modalidad tradicional de educación dada la necesidad de evitar el contacto lo que a su vez previene el contagio, por tanto, la medida de evitar la presencialidad responde a cuidar el derecho a la salud a la vez que se garantiza el derecho a la educación. Por su parte la temporalidad quiere cuidar que aquellas medidas excepcionales, no sean tomadas sino por un tiempo que permita buscar estrategias para, dentro de las posibilidades, poner en vigencia el máximo de derechos al mismo tiempo.

Por su parte, el Libro I de Ley N 1680/2001 que aprueba el Código de la Niñez y la Adolescencia (en adelante CNA), Título Único, Capítulo Único "De las obligaciones de los Estados y de los particulares" se incorpora en el Art. 20 que prevé el derecho a la educación, como aquel que garantiza el desarrollo armónico e integral de la persona y la prepara para el ejercicio de la ciudadanía, coincidiendo in fine con lo expresado por el Art. 5 de la CDN, en cuanto establece que el fin de la protección es la autonomía de la persona.

Así también, la Ley General de Educación, que en el Art. 5 establece 
Sanabria Moudelle, C., \& Velázquez Romero, E. Medidas educativas dispuestas en Paraguay en tiempos de pandemia desde la perspectiva de los derechos de la niñez y la adolescencia

la necesidad de contar con un currículo único, que podría ser adaptado a las necesidades particulares.

Igualmente, reconociendo la necesidad de tener en cuenta a las niñas, niños y adolescentes con discapacidad, se requiere tener presente el Art. 24 de la Convención de las Naciones Unidas por los Derechos de las Personas con discapacidad aprobada por Ley $\mathrm{N}^{\circ} 3540 / 2008$, que establece a cargo de los Estados la obligación de efectivizar este derecho y en especial:

c) Se hagan ajustes razonables en función de las necesidades individuales; d) Se preste el apoyo necesario a las personas con discapacidad, en el marco del sistema general de educación, para facilitar su formación efectiva; e) Se faciliten medidas de apoyo personalizadas y efectivas en entornos que fomenten al máximo el desarrollo académico y social, de conformidad con el objetivo de la plena inclusión. (Ley N³540/ 2008, Art. 24)

Pasamos así en los siguientes puntos a presentar las principales observaciones al Plan de acuerdo a los principios orientadores que la CDN plantea y las tensiones o vulnerabilidades que se generan.

\section{LA INTEGRALIDAD DE LA PROPUESTA EDUCATIVA}

Analizando las estrategias del plan desde la perspectiva de los derechos de la niñez y la adolescencia, tomamos como punta pié inicial, la pregunta sobre cuál es la visión que incorpora el plan. Si se mira a la infancia o a las infancias, considerando desde esta última perspectiva a la diversidad que encontramos en la población. Ante esta pregunta, coincidimos con la afirmación de Gaitán (2017) que explicaba:

La utilización del plural en la palabra infancia quiere subrayar el hecho de que, en la sociedad en la que vivimos, hay muchas formas, distintas, de vivir la infancia, por más que todas las personas que transitan por ella compartan unas características comunes, precisamente por su posición generacional, diferente (vamos solo a decir) a la de la generación adulta. (p. 15)

Complementando esta mirada a las infancias, desde una visión diversa, flexible, abierta, hay que apuntar el otro componente fundamental que debe estar presente para hablar desde el enfoque de derechos. Es pues indispensable, partir de la idea del niño como Sujeto de Derechos (Cillero Bruñol et al., 1997) titulares y merecedores de protección:

desde la verdadera vigencia de la Doctrina de la Protección Integral, deberían ser vistos como actores sociales cada vez más visibles que participan y son parte de la vida al igual que los adultos. Son así mismo, un grupo social, una categoría social. (Ravetllat Ballesté, \& Sanabria Moudelle, 2020, p. 29)

A decir de Ravetllat Ballesté (2011), "La infancia es, sin duda, en nuestra 
conceptualización actual un periodo válido por sí mismo"(p. 51).

Igualmente Ferran Casas (2006), respecto a las representaciones sociales sobre la infancia y su valor, afirma: "Buena parte del valor social de la infancia está en el futuro: son los futuros adultos, los futuros ciudadanos, nuestra sociedad del futuro (moratoria social). No resulta, pues, fácil, pensarlos como ciudadanos del presente" (p. 40). Podemos afirmar entonces que, siendo sujetos de derecho, es una cuestión de justicia y de efectividad del marco jurídico, verlos como ciudadanos del presente, que deben gozar ahora de sus derechos y no constituir una mera expectativa.

Por tanto, para afirmar que el plan efectivamente reconoce a las infancias y las adolescencias y que si valora al conjunto de niñas, niños y adolescentes como categoría social en consonancia con el principio de autonomía progresiva, ello debe reflejarse tanto en su discurso teórico como en su operatividad práctica, sobre el punto, nos dice Latapi Sarre (2009):

Es relativamente fácil puntualizar teóricamente las obligaciones del Estado respecto al Derecho a Educación; su cumplimiento en la práctica, sin embargo, estará condicionado por la viabilidad política y financiera de sus decisiones y por la necesidad de priorizar unas obligaciones respecto a otras. (p. 255-287)

Es decir, la viabilidad política de una propuesta gubernamental es la que condicionará finalmente si se pone en práctica o no acciones que garanticen el acceso a la educación con enfoque de derechos, con lo cual solo se podrá llegar a afirmar que si se observa que el plan educativo incluye a todos y cada uno de los niños, niñas y adolescentes en la diversidad que los caracteriza como grupo social, si en la práctica se los trata con autonomía, ¿̇ y qué implica tratar con autonomía?

Como bien lo señala Gómez De La Torre (2018):

la autonomía progresiva de un menor debe ser evaluada caso a caso, cada niño es único, tomando en cuenta su grado de madurez tanto psíquica como social y culturalmente. Utilizando como parámetros para la evaluación: la familia, el lugar en que vive (no es lo mismo un niño que crece en la capital que un niño que crece en el campo lejos de todo desarrollo tecnológico), la educación, el medio en el que se desarrolla y la situación económica. (p. 4)

Resulta pertinente la observación de la autora supra mencionada en cuanto a que la misma agrega como parámetros para evaluar la autonomía progresiva del niño a: a) la familia, b) el lugar en que vive c) el medio en el que se desarrolla y d) la situación económica.

En esa misma línea, La Comisión Interamericana de Derechos Humanos (CIDH), Abril 2020 en su Comunicado de Prensa N 90/20, recomienda a los Estados:

3. Disponer de mecanismos que permitan a los NNA seguir con el acceso a la educación y con estímulos que su edad y nivel de desarrollo requieran. (CIDH, 2020a, para. 14) 
Por tanto, si en la ejecución del plan se descuida o no se considera atención diferenciada y ajustes según los parámetros citados difícilmente puede hablarse de cumplimiento del principio de autonomía progresiva. Es decir reconocer y respetar el principio de autonomía progresiva implica necesariamente ofrecer una propuesta educativa integral que atiende a las necesidades del cien por ciento de la población de niñas, niños y adolescentes en edad escolar (currículo basado en competencias mínimas para el año, condiciones socioeconómicas, etapa evolutiva de la niña, niño, adolescente).

Al respecto, el Plan (MEC, 2020) hace ciertas distinciones, por ejemplo bien considerando lo que denomina "casos especiales" (pp. 18-20) mencionando a la población indígena, a la niñez con necesidades específicas de apoyo educativo, las aulas hospitalarias, entre otros, sin embargo, no llega a profundizar justamente en esos parámetros más pertinentes a este contexto que el lugar en que vive y la situación económica, lo cual puede causar tropiezos o tensiones para poder afirmar, en el futuro que el plan tiene un enfoque que considera el niño como sujeto de derechos.

Tampoco se hace referencia a la necesaria Inter institucionalidad que resulta necesaria para trabajar de cerca con determinados sectores de la niñez y adolescencia y para lo cual se necesita coordinación con la Secretaría Nacional por los Derechos de la Personas con Discapacidad (SENADIS), El Ministerio de la Niñez y la Adolescencia, el Instituto Paraguayo del Indígena, el Ministerio de Desarrollo Social, el Ministerio de Justicia.

A modo de ejemplo en cuanto a la necesaria articulación, para lograr un abordaje más integral y con alcance a nivel nacional se podría mencionar la necesidad de trabajo inter institucional entre el MEC y la SENADIS para el fortalecimiento de la aplicación de la Ley de Educación Inclusiva en atención a las personas con discapacidad, en línea con lo recomendado por la Comisión Interamericana de Derechos Humanos en su reciente resolución: Asegurar que las niñas y los niños con algún tipo de discapacidad, puedan acceder a la educación en línea sin exclusiones, mediante sistemas de apoyo, estrategias de comunicación y contenidos accesibles (Resolución 1 CIDH, 2020b). Como decía Villagrasa (2009) respecto a la integración de las acciones:

Se trata de una respuesta global, que supere las iniciativas sectoriales, identificadas con derechos concretos, que se llevan a cabo desde la perspectiva asistencial de cubrir necesidades. El reto de la Convención está en garantizar un entorno que garantice el ejercicio y exigibilidad de los derechos del a infancia y la adolescencia. (p. 56)

Parece atinado, mencionar que el modelo educativo venía mostrando dificultades para ser considerada como universal y pertinente, inclusiva si se quiere, pensando en las infancias que están indudablemente conformadas por 
una inmensa diversidad. Esta idea es abordada por Demelenne (2018) que, si bien contextualiza su análisis en la educación intercultural, nos da pistas para pensar en una verdadera educación inclusiva y afirma que lo que se requiere es un cambio estructural que pase por la experiencia y no meramente por el hecho de "impartir" conocimientos:

Cuando hablamos de nuevos marcos referenciales, es reconocer que no sabemos como hacerlo y menos como capacitar a los docentes para hacerlo, tampoco sabemos si los espacios y los actores educativos actuales son los más pertinentes. Una nueva educación indígena, pero también una nueva educación inclusiva (integrando todas las formas de diversidades) no necesita un cambio curricular sino de una trasformación estructural. (p. 150)

Englobando las ideas antes expuestas, creemos pertinente acompañar la reflexión del maestro Tonucci (2020) realizada en el Seminario denominado "Por una buena escuela en tiempo de pandemia", en el cual manifestaba que son dos las barreras que debe superar la educación: la primera, la enseñanza centrada en culminar el programa de estudio y no en el desarrollo de la personalidad del niño; y, la segunda, la confrontación entre la escuela y la familia, siendo indispensable, la unión de ambas como entorno privilegiado del niño.

En conclusión, consideramos necesario tener en cuenta ciertos aspectos que permitirán integrar el enfoque de derechos al plan nacional de educación en tiempos de pandemia, hemos apuntado entre ellos: la ausencia de una consideración del niño como sujeto de derechos; la mirada a homogénea no orientada a la diversidad, a las infancias; no considerarlos como presente; poner el énfasis en el desarrollo del programa y no de la personalidad del niño como manda la CDN; ausencia de articulación entre los actores y sectores; no reconocimiento de las competencias que demuestran las niñas, niños y adolescentes para proponer respuestas desde la pertinencia ajustada al desarrollo evolutivo.

Por su parte, la falta de involucramiento de otras instancias estatales que también tienen como beneficiarios al sector de la niñez y la adolescencia, para implementar las estrategias, obstaculiza que el principio de autonomía progresiva efectivamente se consolide en la ejecución del plan y que la propuesta en si tenga un abordaje integral.

\section{LA BRECHA DIGITAL COMO REALIDAD REGIONAL Y NACIONAL QUE CONDICIONA EL EJERCICIO EFECTIVO DEL ACCESO A LA EDUCACIÓN}

El Plan MEC 2020 propone principalmente el desarrollo de las clases en la modalidad virtual. Esta premisa implica cumplir tres requisitos fundamentales para que el proceso de enseñanza aprendizaje pueda llevarse a cabo sin mayores inconvenientes: a) acceso a internet; b) conectividad óptima 
Sanabria Moudelle, C., \& Velázquez Romero, E. Medidas educativas dispuestas en Paraguay en tiempos de pandemia desde la perspectiva de los derechos de la niñez y la adolescencia

a internet; $y$ finalmente, $c$ ) docentes con competencias digitales para enseñar en esa modalidad virtual.

De la clasificación presentada previamente puede visualizarse por un lado que este artículo propone diferenciar como categorías autónomas el "acceso a internet" de la "conectividad óptima internet" en el entendimiento de que acceder efectivamente al uso de las tecnologías de la información y comunicación con las bondades y beneficios que ofrece para el proceso educativo va más allá de contar con un dispositivo de acceso a internet sino que ese acceso sea en condiciones óptimas tales que permita que el usuario beneficiario pueda utilizar todas las herramientas y recursos a disposición. La noción sale justamente a partir del sonado concepto de "brecha digital".

Sobre el concepto de "brecha digital" podemos recurrir a lo que nos dice Claflin (2000) "la separación que existe entre las personas que utilizan las nuevas tecnologías de la información como una parte rutinaria de sus vidas y de aquellas que no tienen acceso a las mismas y aunque las tuvieren no saben cómo utilizarlas"; en igual sentido Serrano Santoyo y Martínez (2003) hablan de una "desigualdad de posibilidades que existen para acceder a información, conocimiento y educación mediante las tecnologías de la información y comunicación" (p.17), continuan señalando los mismos autores que la "brecha digital" va más alla de la falta de acceso meramente sino que combina otros factores sociales y económicos.

Estas desigualdades mencionadas precedentemente que hacen al concepto de "brecha digital" son una realidad en nuestro país, de hecho en el propio documento del Plan MEC 2020 se reconoce que conforme a datos actualizados provistos por la Dirección General de Estadísticas y Censos el $24,4 \%$ de los estudiantes cuentan con acceso a internet de banda ancha y 24 , $6 \%$ dispone de una notebook según el mismo plan describe (MEC, 2020, p. 13) un porcentaje demasiado bajo para hablar de una política que debería ser universal. Por otro lado, el documento afirma que la propuesta está centrada en la oferta del servicio educativo adaptado al teléfono dado que la mayor parte de la población de 5 a 17 años tiene acceso a un celular (MEC, 2020, p 14).

Esta realidad no se aleja mucho de lo que ocurre a nivel regional, como refiere CEPAL (2019) y autores como Trucco y Palma (2020) que si bien se han logrado avances en cuanto a reducir las brechas de acceso al mundo digital gracias a existe un mayor acceso a la conectividad móvil, aún persisten brechas considerables en el acceso efectivo al mundo digital.

Es por ello que la estrategia de la virtualidad presentada en el plan debe necesariamente complementarse con otras variantes, en las cuales se combine la virtualidad con el acompañamiento, más aun teniendo en cuenta la información antes citada que describe a una población en su mayoría sin acceso a las tecnologías. A la vez, se podría haber incorporado como prioridad que esa educación sea pertinente, flexible, individualizada, accesible, gratuita. Si bien se 
menciona la modalidad no virtual, delimita en cuanto a sus beneficiarios y no especifica acciones concretas de llegada.

Sumado a esto, vemos el tercer requisito propuesto que es de las competencias digitales docentes, entendiendo estas como "el conjunto de conocimientos y habilidades necesarios que se deben poseer para utilizar estas herramientas tecnológicas como unos recursos educativos más integrados en su práctica diaria" (Suárez-Rodríguez et al., 2012) o como bien señala el propio Parlamento Europeo en una recomendación publicada ya en el año 2006:

La competencia digital implica el uso crítico y seguro de las Tecnologías de la Sociedad de la Información para el trabajo, el tiempo libre y la comunicación. Apoyándose en habilidades TIC básicas: uso de ordenadores para recuperar, evaluar, almacenar, producir, presentar e intercambiar información, y para comunicar y participar en redes de colaboración a través de Internet (Unión Europea, 2006, p. 6).

Es decir, el docente como actor clave en el proceso educativo necesariamente debe contar con las competencias necesarias para dar un correcto uso a las tecnologías de la información y comunicación y generar verdaderos entornos virtuales de aprendizaje. Se genera así la necesidad de contar con la cantidad suficiente de docentes con competencias digitales.

En conclusión, la "brecha digital" existente a nivel regional y puntualmente en Paraguay afecta el proceso educativo y el acceso efectivo a educación de las niñas, los niños y adolescentes, con lo cual, si se pretende desarrollar políticas educativas que prioricen el interés superior del niño se debe pensar en políticas públicas macro a nivel intersectorial que apunten a facilitar el acceso a buena conectividad de internet y considerar incluso porque no, el internet como un servicio público, encontrándose suficiente argumento justificante en la propia $\mathrm{CN}$ que reconoce el interés superior del niño. Dicho principio debe priorizarse y anteponerse para que la niñez y la adolescencia desarrolle su proceso educativo con las herramientas necesarias, en esta situación: buen acceso y conectividad a internet. Igualmente, contar con la herramienta en sí no es suficiente, los actores claves del proceso educativo, los docentes, deben contar con la formación necesaria en competencias digitales, se caería en un error en priorizar solo la conectividad y descuidar al personal docente.

En Paraguay no existen datos suficientes sobre de la totalidad de docentes matriculados y activos cuantos cuentan con competencias digitales, esta información es más que necesaria.

\section{EL ROL DE LAS FAMILIAS Y LAS ESTRATEGIAS DE EMPODERAMIENTO Y FORTALECIMIENTO TENIENDO EN CUENTA QUE EL ÉXITO DE LA POLIITICA EDUCATIVA DESCANSA EN ELLA}

Ahora bien, al inicio de este artículo se hacen unas preguntas iniciales que planteaban las problemáticas a ser analizados, una de esas preguntas iba relacionada con las familias. ¿Cómo se proporciona a las familias, las condiciones 
Sanabria Moudelle, C., \& Velázquez Romero, E. Medidas educativas dispuestas en Paraguay en tiempos de pandemia desde la perspectiva de los derechos de la niñez y la adolescencia

para apoyar el proceso educativo? ¿Cómo se contempla el acompañamiento presencial a las familias en las comunidades desde el inicio de la crisis sanitaria? Sobre todo entendiendo que, en contextos vulnerables, ¿es más efectiva la llegada presencial?

Surge el planteamiento del apoyo y acompañamiento a las familias ya que la situación que se vive exige una mayor asistencia a las familias. En el plan se establece la aplicación de una encuesta para brindar apoyo a aquellas que lo necesiten, sin embargo, la propuesta no plantea una solución práctica, no se describe con claridad de qué manera se logrará esa llegada. Al parecer estaría ausente la previsión universal, una propuesta para todas las familias. El hecho de haber cambiado de modalidad y pasar a la virtualidad-sin precedentes en la historia de esta generación- hace que se deba proponer un acompañamiento a todas y cada una de las familias, para comprender y adaptarse a este nuevo proceso. Se observa la necesidad de prever un proceso de adaptación a esta nueva forma de vivir. Puede decirse que en este periodo de pandemia el Estado y la política pública descansan sobre las familias, toda la responsabilidad en este periodo está puesta en ella.

En el apartado VII "Orientaciones a las familias" (pp. 31-36) inciso e, se establece que las familias deberán brindar contención a los niños, niñas y adolescentes. No obstante, ello, no queda claro de qué manera se fortalecerá a las familias para que cuenten con las competencias necesarias vinculadas a este requerimiento.

En el Plan MEC 2020 se establecen distintas obligaciones que están bien enunciadas, incluso es posible notar su correspondencia con el modelo de disciplina positiva que incorpora la Ley $N^{\circ} 5659 / 2016$, Promoción del buen trato, crianza positiva y de protección a niños, niñas y adolescentes contra el castigo físico o cualquier tipo de violencia como método de corrección o disciplina, sin embargo no se prevé la forma de hacer operativas estas premisas.

En este sentido, la CIDH (2020a) en su recomendación publicada señala:

4. Promover rutinas de actividades para niñas, niños y adolescentes, brindando herramientas para que las familias puedan propiciar actividades recreativas y juegos que les permitan momentos de esparcimiento, a los fines de garantizar la salud física, psíquica y emocional de los NNA. (CIDH,2020a, para. 15)

Esta propuesta de la $\mathrm{CIDH}$ da pie a considerar, porque no, lo que se conoce como "enfoque centrado en la familia" muy utilizado sobre todo en educación inicial pero que presenta herramientas, técnicas y sobre todo un abordaje que bien podría replicarse a otros niveles educativos. Al respecto:

El enfoque centrado en la familia reconoce que cada familia es única; que la familia es la constante en la vida del niño; y que ellos son los expertos en las capacidades y necesidades de los niños. La familia trabaja con los profesionales para tomar decisiones informadas sobre los servicios y apoyos que el niño y 
la familia reciben. En la intervención centrada en la familia, se consideran las fortalezas y necesidades de todos los miembros. (Law et al., 2003, p. 2)

Es interesante dicho enfoque por cuanto reconoce al niño en su entorno natural que es su familia y en cierta forma obliga a un mayor acercamiento por parte de las instituciones educativas pero más que nunca ese acercamiento se necesita tomando en cuenta el nuevo rol que toca a los padres hoy asumir. En ese sentido, como nos dice Dalmau (2017):

Pone énfasis en entender el desarrollo del niño de una manera más holística y más contextual; se reconoce que el progreso del niño no responde a una suma de intervenciones parciales sino a una visión de conjunto que encuentra la mejor expresión en la atención preferente en casa y la comunidad. (p. 642)

Resaltamos de lo citado, la mirada contextual que este modelo propone y que debe ser el enfoque de la actual política educativa, con acciones congruentes de la propia realidad nacional con las dificultades propias que tienen las familias y están atravesando actualmente.

Es posible tener como referencia, lo señalado en el reciente informe CEPAL-UNESCO (2020) sobre educación y pandemia citando a su vez al informe hecho por CEPAL y UNICEF (2020):

El 51,2\% de niñas, niños y adolescentes que viven en zonas urbanas en América Latina reside en hogares con algún tipo de precariedad habitacional. Dos de cada diez viven en condiciones de precariedad habitacional moderada y tres de cada diez enfrentan situaciones de precariedad habitacional grave. (CEPAL \& UNICEF, 2020 citado en CEPAL \& UNESCO, 2020, p. 13)

La precariedad habitacional en tan alto porcentaje que estos informes señalan no es menor y repercute lógicamente en el desarrollo de las niñas, niños y adolescentes. Otros datos preocupantes que muestran los citados informes:

La investigación ha demostrado la importante relación que existe entre las privaciones en el contexto habitacional y la vulneración de otros derechos de la infancia. El hacinamiento impide contar con un espacio adecuado para estudiar y descansar, lo que repercute en el desarrollo cognitivo en la infancia y las trayectorias laborales y de bienestar en la adultez, a la vez que favorecen una mayor propensión a situaciones de abuso (CEPAL/UNICEF, 2020, p. 14).

Es decir, entre precariedad habitacional y vulneración de derechos hay una conexión directa y lamentable que se presta a todo tipo de abusos que inciden, sin duda el proceso educativo, por ende, un plan educativo oportuno sería aquel que se involucre y comprenda el real contexto del niño en su entorno, resaltando nuevamente el modelo centrado en la familia.

Surge igualmente la interrogante de cómo aplicar este modelo, si es factible o no. En ese sentido, García-Sánchez et al. (2014), mencionado a su 
Sanabria Moudelle, C., \& Velázquez Romero, E. Medidas educativas dispuestas en Paraguay en tiempos de pandemia desde la perspectiva de los derechos de la niñez y la adolescencia

vez a otros autores señalan algunas estrategias sencillas de aplicación que confirman la factibilidad de adaptar el modelo educativo a un enfoque centrado en la familia, asi:

a. Fomentar el intercambio de conversación e información con la familia. b. Observar (en el entorno natural). c. Demostrar la intervención. d. Enseñar a los padres directamente. e. Dar feedback a los padres sobre sus prácticas. f. Desarrollar una interacción conjunta. g. Proporcionar guía práctica con retroalimentación. h. Utilizar técnicas de resolución de problemas. i. Centrar objetivos en el niño (Rush \& Shelden, 2011; Stoner et al., 2013; Meadan \& Angell (2013) citados en García-Sánchez et al., 2014).

Muchas de estas estrategias citadas se han logrado con el proyecto denominado "Maestras Mochileras" impulsado en su momento gracias a la cooperación internacional por parte de UNICEF en Paraguay. El proyecto en sí puede ser replicado, con todas las medidas de protección y seguridad en este contexto sobre todo en las poblaciones vulnerables, de hecho figura en el Plan de Acción Educativa 2018-2023 (MEC, 2018) y cuenta con una recomendación por la OECD (2018) de que continúe.

En conclusión, para este apartado y como se visualiza todas las estrategias plantean colocar al niño en el centro de atención y a su entorno familiar, lo que contribuirá para que la política educativa brinde una respuesta más apropiada.

\section{EL SISTEMA DE EVALUACIÓN}

En cuanto a la evaluación, en el Plan MEC 2020, menciona que será procesual y que el docente hará una valoración tanto cualitativa como cuantitativa afirmando que el contenido que requiera refuerzo se desarrollará en el año 2021. Sobre el punto se debe tener en cuenta que, por un lado, no hay certeza del retorno a la normalidad y por otro, si se apunta a recuperar o reforzar contenidos el año siguiente, se debería prever una mirada integral a las necesidades y derechos de las niñas, niños y adolescentes.

El Plan se complementa a su vez con la Resolución N 602/2020 "Por la cual se aprueba el documento Orientaciones para evaluar el aprendizaje de los estudiantes en los diferentes niveles y modalidades del Sistema Educativo Nacional en tiempos de pandemia".

En la Resolución 602/2020, se menciona que la evaluación es formativa, que servirá-resumiendo lo expresado en la norma- para indagar qué está funcionando y que debe ser reorientado dentro del proceso de aprendizaje. Este enunciado, pareciera tener poca relación con el plan establecido, habida cuenta que, no se menciona de qué manera se darán los ajustes en caso de que sea necesario.

El Comité de los Derechos del Niño, en la Observación General N 1 sobre los Propósitos de la Educación, y particularmente en lo que respecta a 
la evaluación, hace énfasis no solo, sino la propuesta educativa en su conjunto para comprender si se ajusta o no a la efectividad del derecho. Se hacía mención también de que en ese proceso de evaluación deberían participar todos los actores involucrados, incluidas las niñas y niños:

Todo niño tiene derecho a una educación de buena calidad, lo que a su vez exige concentrar la atención en la calidad del entorno docente, de los materiales y procesos pedagógicos, y de los resultados de la enseñanza. El Comité señala la importancia de los estudios que puedan brindar una oportunidad para evaluar los progresos realizados, basados en el análisis de las ideas de todos los participantes en el proceso, inclusive de los niños que asisten ahora a la escuela o que ya han terminado su escolaridad, de los maestros y los dirigentes juveniles, de los padres y de los supervisores y administradores en la esfera de la educación. (CRC/GC/2001/1, Abril de 2001, párrafo 22)

En la evaluación se contempla la posibilidad de que el alumno que no supere o apruebe todas las áreas académicas, tendrá derecho a las estrategias de promoción asistida y nivelación del aprendizaje. Estas estrategias parecen menos practicables en el entorno virtual, no solo por el requisito de acompañamiento cercano que requerirá-siendo de difícil cumplimiento en la virtualidad-sino también teniendo en cuenta que, al parecer la Pandemia aún no ve un final cercano por lo que, como mínimo se plantea: ¿Qué pasará si en el 2021 se ve la necesidad de continuar con las clases en modalidad virtual? La cohorte 2020, por el momento ve comprometida seriamente sus competencias.

Se debe mirar con atención igualmente la propuesta que se plantea para aquellos alumnos que no puedan acceder a ser promovidos, y por el contrario, debían hacer la nivelación. En ese caso, se plantea que el estudiante cursará durante el año lectivo, en un turno el grado que le corresponde y en el turno opuesto, las materias que no haya superado. Esta propuesta nuevamente carece de un criterio práctico en cuanto a dedicación, acompañamiento-más aún si se espera que la familia esté durante toda la jornada-, condiciones materiales, y lo que debería ser el centro: ¿Cuántas horas debería estar una niña, niño o adolescente frente a la pantalla?

Quizás podría servirnos de orientación para proyectar el impacto de la pandemia, el resultado de la Encuesta de Percepción aplicada a las familias, en la cual se ha consultado sobre "¿Cuánto te parece que la situación generada por la COVID-19 les afecta a vos y a tu familia?" a la cual los encuestados manifestaron en un $87 \%$ sentirse afectados en lo educativo, seguido de un $85 \%$ en lo emocional y un $84 \%$ en lo psicológico (UNICEF, 2020, p. 17). Se mencionan estos dos aspectos del bienestar dado su impacto directo en el entorno del niño. 
Sanabria Moudelle, C., \& Velázquez Romero, E. Medidas educativas dispuestas en Paraguay en tiempos de pandemia desde la perspectiva de los derechos de la niñez y la adolescencia

Como bien señala el reciente informe publicado por CEPAL y UNESCO (2020):

no existen soluciones universales si existen ciertas consideraciones mínimas como ser: a) sopesar la oportunidad y la utilidad de la evaluación para proporcionar retroalimentación a las y los estudiantes, y monitorear sus aprendizajes y los efectos de las estrategias desplegadas en el contexto actual, con miras a la reapertura de escuelas; b) buscarse mecanismos que aseguren la equidad del proceso evaluativo, considerando que la actual crisis está afectando diversos aspectos de la preparación del alumnado para estos exámenes, incluidos el progreso en los aprendizajes, la disponibilidad de infraestructura e incluso el estado y el desarrollo de las habilidades socioemocionales. (p. 9)

Es decir, aquí nuevamente entra en consideración la mirada desde el principio de la autonomía progresiva y la consideración integral de la niñez y la adolescencia.

Sobre el punto coincidimos con autores como Sosa (2020) quien sugiere la idea de priorizar el "el aprendizaje por sobre la evaluación" (p. 6), es decir asegurarse que la niñez y adolescencia efectivamente acceda a educación de calidad, disfruten el proceso y no se asuma la evaluación como un elemento desalentador.

\section{LA PARTICIPACIÓN DE LOS PROPIOS NIÑOS, NIÑAS Y ADOLESCENTES EN LA PROPUESTA EDUCATIVA}

Como afirman Matos Lins (2020), en el artículo titulado Derecho a la participación política de la infancia en la escuela: Algo cambiará en función a la pandemia?

Da forma como compreendemos, os acontecimentos afeitos à relação das crianças e seus processos de esco-larização estão sendo engendrados sem a devida preocupação sobre a dimensão geracional, por parte dos adultos envolvidos (familiares e profissionais e pelos que realizam as políticas públicas). Nesse contexto, os órgãos gover-namentais decretam sobre a vida, também escolar, dessas crianças e jovens, sem consultar os principais envolvidos. (Gaitán, 2017, p. 247)

Los verdaderos destinatarios de las acciones educativas están siendo ignorados en su calidad de ciudadanos de hoy, del presente, como parte del proceso que viven y como merecedores de su propio protagonismo y del derecho que la ley les consagra: la participación en todos los asuntos que les compete. ¿¿es compete su educación? La respuesta parece unánime, el propio fin de la educación desde un paradigma de vanguardia debería promoverla. Por tanto, consideramos que cualquier acción que se emprensa debe contar con la voz y participación protagónica de las niñas, niños y adolescentes.

Se conoce ya alguna experiencia de participación en los procesos de la política pública en Paraguay, se destaca, así como positivo la iniciativa dispuesta 
por el MEC de realizar las reuniones virtuales denominadas Aty Guasu en guaraní iniciadas en mayo de 2020 (ABC, 2020), esta propuesta por lograr el consenso educativo en la construcción de políticas educativas no obstante debe ser la regla y no la excepción.

Es ahora el momento de tener presente estas iniciativas y emularlas a fin de que aquel principio tan invocado de la participación se haga realidad. Volvemos a insistir igualmente en que solo así se podría dar un proceso de incorporación de la ciudadanía activa y cumplimiento de los fines de la educación, cuál debe ser el desarrollo de la personalidad de la niña, niño y adolescente.

Como bien nos sugiere Romero de Velázquez (2011), resaltando la idea de que las políticas educativas deben involucrar a la ciudadanía toda, reflexiona la autora diciendo que "este es el tiempo de recuperar nuestra soberanía nacional y nuestra dignidad, asumiendo el compromiso de sumar nuestras diferencias y delinear juntos un nuevo pacto social que nos permita construir un "nosotros", participativamente, con absoluta autonomía y responsabilidad social" (p. 124).

Coincidimos con la autora en cuando que justamente la participación efectiva de las niñas, niños y adolescentes en la construcción, ejecución y evaluación de las políticas educativas consolidarán el pacto social tan necesario para llegar al anhelado bienestar común y en este caso en particular, acceso efectivo a educación.

\section{Conclusiones y Recomendaciones}

Del análisis que antecede es posible deducir que, por un lado se cuenta con un plan concebido desde una perspectiva aun teórica en la que se lee poco sobre las estrategias o acciones reales para llegar a toda la población (la política de educación debe ser universal) y por otro lado, que fue concebida más bien pensando en un modelo de niñez y de adolescencia (aquella que sea capaz de estar frente a las pantallas determinadas horas, siguiendo el programa establecido). Tampoco es clara en cuanto al apoyo efectivo que se brinda a las familias, aun siendo ella donde descansa el éxito de este periodo (las familias están cumpliendo el rol que antes hacia la escuela).

Estamos convencidos que, si la escuela va a la casa, la casa debería estar preparada. Según se verifica en el Plan, el mismo se establece con el objetivo de garantizar el derecho a la educación centrando la propuesta en facilitar el proceso enseñanza- aprendizaje. Tanto el Plan como la Resolución $N^{\circ} 602$, se constituyen en instrumentos normativos algo retóricos, enunciativos, teóricos, al expresar una serie de estrategias que están desvinculadas con la evidencia principal que cita el mismo documento cuando menciona que tan solo el $24,4 \%$ de la población cuenta con internet de banda ancha.

Se concluye que el plan fue concebido como teórico y no contempló 
estrategias de llegada efectiva al universo de la población; las estrategias de contacto con las familias fueron inexistentes; la propuesta educativa no se planteó flexible e individualizada; la evaluación se enfocó en el rendimiento y no el desarrollo de la personalidad; se observaron serias limitaciones en cuanto al acceso a internet y formación del docente; no se consideró la autonomía y participación de los principales destinatarios de la política.

El estudio permitió igualmente ofrecer recomendaciones para mitigar las posibles vulneraciones del derecho a la educación, para nosotros, sostener el derecho a la educación en el contexto paraguayo implicaría:

1. Generar estrategias de llegada a las familias. Realizar encuentros al menos quincenales en espacios abiertos, con grupos pequeños. La socialización es clave sobre todo para el nivel de educación inicial y la escolar básica.

2. Diseñar planes flexibles e individualizados que consideren a las infancias y a las adolescencias. Se deberán adoptar las estrategias del modelo/enfoque centrado en la familia ayudando a lograr una verdadera conexión entre sistema educativo y comunidad/familias, mitigando a la vez que aumenten o se acentúen vulneraciones al niño, niña y adolescente dentro su propia casa. Considerar en esta línea experiencias de llegada a las familias como el proyecto de "maestras mochileras"

3. Enfocar las estrategias educativas en el desarrollo de la personalidad del niño y no solo en seguir el programa o lo que es peor, finalizar el programa previsto para el año académico.

4. Disminuir la brecha digital concebida no solo desde la perspectiva de la accesibilidad y conectividad a internet sino además el fortalecimiento de la capacitación docente en competencias digitales.

5. Brindar acompañamiento socio-emocional a familias y docentes.

6. Fortalecer el trabajo interinstitucional intersectorial entre organismos del Estado sobre todo aquellos directamente involucrados en el ámbito de niñez y adolescencia, con acciones en este escenario: SENADIS, MITICS, MINNA.

7. Aplicar el enfoque integral a la propuesta educativa, considerando el principio de la autonomía progresiva; teniendo como centro a la niñez y la adolescencia.

8. Asegurar la participación protagónica de niñas, niños y adolescentes durante la pandemia para la respuesta; y en la planificación de acciones para la post pandemia. 


\section{REFERENCIAS}

ABC Color. (27 de mayo de 2020). MEC realiza "aty guazú" virtual este jueves. https://www.abc.com.py/edicion-impresa/locales/2020/05/27/ mec-realiza-aty-guasu-virtual-este-jueves/

Casas, F. (2006). Infancia y representaciones sociales. Instituto de Investigaciones sobre Calidad de Vida. Universidad de Girona. Revista Políticas y Sociedad, 43(1): 27-42. https://www.researchgate.net/ publication/27591450_Infancia_y_representaciones_sociales

CEPAL, \& UNESCO. (2020). La educación en tiempos de la pandemia de COVID-19 - Informe CEPAL-UNESCO COVID-19". https://unesdoc. unesco.org/ark:/48223/pf0000374075 ? posInSet $=1 \&$ queryld $=660$ 6d041-e555-4f06-b4c4-42ea1b4153e9

CEPAL, \& UNICEF. (2020). La ciudad y los derechos de niñas, niños y adolescentes. Desafios, (23).

Cillero Bruñel, M. (1997). Infancia, autonomía y derechos: una cuestión de principios. http://www.iin.oea.org/Cursos_a_distancia/explotacion_ sexual/Lectura4.Infancia.DD.pdf

Claflin, B. (13 de octubre de 2000). Artículo de Prensa Publicado "EL ABC y D de la brecha digital". Diario Reforma, Sección Negocios.

Comisión Económica para América Latina y el Caribe, CEPAL. (2019). Panorama Social de América Latina, 2018 (LC/PUB.2019/3-P).

Comisión Interamericana de Derechos Humanos. (27 de abril de 2020a). Comunicado de Prensa 90/2020. http://www.oas.org/es/cidh/prensa/ comunicados/2020/090.asp

Comisión Interamericana de Derechos Humanos. (2020b). Resolución N 1/20 Pandemia y Derechos Humanos en las Américas. CIDH/OEA. https:// www.oas.org/es/cidh/decisiones/pdf/Resolucion-1-20-es.pdf

Comité de los Derechos Del Niño. (2001). Observación General N 1 Propósitos de la Educación. CRC/GC/2001/1. https://www.unicef.org/UNICEF-Obse rvacionesGeneralesDelComiteDeLosDerechosDelNino-WEB.pdf

Constitución Nacional, 1992.

Convención de las Naciones Unidas sobre los Derechos del Niño, 20 de noviembre de 1989. https://www.ohchr.org/en/professionalinterest/pages/crc. aspx

Dalmau, M. (2017). Cómo implementar el modelo centrado en la familia en la intervención temprana. Anales de psicología, 33(3), 641-651. http:// dx.doi.org/10.6018/analesps.33.3.263611

Decreto $N^{\circ} 3456 / 2020$, Por el cual se declara Estado de Emergencia Sanitaria en 
Sanabria Moudelle, C., \& Velázquez Romero, E. Medidas educativas dispuestas en Paraguay en tiempos de pandemia desde la perspectiva de los derechos de la niñez y la adolescencia

todo el territorio nacional para el control del cumplimiento de las medidas sanitarias dispuestas en la implementación de las acciones preventivas ante el riesgo de expansión del Coronavirus (COVID-19).

Demelenne, D. (2018). Derecho a la Educación desde una perspectiva intercultural, en Nuevas Lecciones para la defensa legal de los derechos humanos de la infancia y la adolescencia en Paraguay. Editora Intercontinental.

Gaitán, L. (2017). Presentación. Revista Sociedad e Infancias. https://revistas. ucm.es/index.php/SOCl/article/view/57270/51607

García-Sanchez, F.A. (2014). Atención Temprana Centrada en la Familia. Revista Española sobre Discapacidad Intelectual, 45(251), 6-27.

Goméz de la Torra, V. (2018). Las implicancias de considerar al niño sujeto de derechos. Revista de Derecho (UCUDAL), 14(18). http://www.scielo. edu.uy/pdf/rd/n18/2393-6193-rd-18-117.pdf

Latapi Sarre, P. (2009). El derecho a la educación: su alcance, exigibilidad y relevancia para la política educativa. Revista Mexiana de Investigación Educativa, 14(40). http://www.scielo.org.mx/scielo. php?script=sci_arttext\&pid=S1405-66662009000100012

Law, M., Rosenbaum, P., King, G., King, S., Burke-Gaffney, J., Moning, J., Teplicky, R. (2003). What is family-centred service? McMaster University.

Ley $N^{\circ}$ 1264/1998, General de Educación.

Ley N¹680/2001, Que aprueba el Código de la Niñez y la Adolescencia

Ley N³540/2008, Que apruebayratifica Convención sobrelos Derechos Humanos de las Personas con Discapacidad y protocolo facultativo.

Ley N5136/2013, De Educación Inclusiva.

Ley $N^{\circ}$ 5659/2016, Promoción del buen trato, crianza positiva y de protección a niños, niñas y adolescentes contra el castigo físico o cualquier tipo de violencia como método de corrección o disciplina.

Ley N57/1990, Que aprueba la Convención de las Naciones Unidas sobre los Derechos del Niño.

Matos Lins, H. (2020). Direito à participação política de crianças sobre a escola: algo mudaria em função da pandemia? Revista Sociedad e Infancias, 4, 243-249. https://revistas.ucm.es/index.php/SOCl/article/ view/69637/4564456554061

Ministerio de Educación y Ciencias del Paraguay. (2020). iTu Escuela en Casa!. https:// aprendizaje.mec.edu.py/aprendizaje

Ministerio de Educación y Ciencias. (2018). Plan de Acción Educativa 2018- 
2023. https://www.mec.edu.py/index.php/es/todas-las-categorias/ category/98-plan-de-acci-n-educativa-2018-2023

Ministerio de Educación y Ciencias. (2020). Resolución N602/2020, Por la cual se aprueba el documento Orientaciones para evaluar el aprendizaje de los estudiantes en los diferentes niveles y modalidades del Sistema Educativo Nacional en tiempos de pandemia.

Ministerio de Educación y Ciencias. (2020). Resolución N³08/2020.

Organización para la Cooperación y el Desarrollo Económico, OECD. (2018). Caminos de Desarrollo Estudio multidimensional de Paraguay. Volumen 2. Análisis detallado y recomendaciones. https://www.oecdilibrary.org/sites/9789264306226-7-es/index.html?itemld=/content/ component/9789264306226-7-es

Ravetllat Ballesté, I. (2011). Marco internacional e interno del Derecho de la Infancia y la Adolescencia" en "Derecho de la Persona. Editorial Bosch. Barcelona.

Ravetllat Ballesté, I., \& Sanabria Moudelle, C. (Eds.) (2020). Autonomía, Infancia y Derechos. Situaciones y cuestiones actuales a 20 años de la aprobación del Código de la niñez y la adolescencia de Paraguay. Universidad Iberoamericana, Editora Intercontinental.

Romero de Velázquez, S. (2011). La educación en el contexto jurídico paraguayo (Tesis Doctoral). Universidad Nacional de Asunción.

Rush, D. D., \& Shelden, M. L. (2011). The Early Childhood Coaching Handbook. Paul H. Brookes.

Serrano Santoyo, A., \& Martinez Martinez, E. (2003). La Brecha Digital: Mitos y Realidades. Editorial de la Universidad Autónoma de Baja California.

Sosa, A. (2020). Educar en tiempos de (pandemias y otras circunstancias). Reflexiones en estado de confinamiento. https://postgrado.unibe.edu. py/images/2020/Educar_en_tiempos_de_confinamiento_y_otras_ circunstancias.pdf

Stoner, J., Meadan, H., \& Angell, M. (2013). A model for coacching parents to implement teaching strategies with their young children with language delay or developemtal disabilities. Perspectives on Language Learning and Education, 20, 112-119.

Suárez-Rodríguez, J. M., Almerich, G., Díaz-García, I. \& Fer Nández-Piqueras, R. (2012). Competencias del profesorado en las TIC. Influencia de factores personales y contextuales. Universitas Psychologica, 11(1), 293-309. http://goo.gl/VCz6jD 
Sanabria Moudelle, C., \& Velázquez Romero, E. Medidas educativas dispuestas en Paraguay en tiempos de pandemia desde la perspectiva de los derechos de la niñez y la adolescencia

Tonucci, F. (25 de abril de 2020). Por una buena escuela en tiempo de pandemia. Seminário online Integratek.

Trucco, D., \& A. Palma (Eds.) (2020). Infancia y adolescencia en la era digital: un informe comparativo de los estudios de Kids Online del Brasil, Chile, Costa Rica y el Uruguay. Documentos de Proyectos (LC/TS.2020/18). Comisión Económica para América Latina y el Caribe (CEPAL).

UNICEF. (2020). Conocimientos, actitudes y prácticas de comportamiento de familias paraguayas sobre la COVID-19: resultados de la encuesta. 2020. https://www.unicef.org/paraguay/media/5431/file/Informe\%20 del\%20Estudio\%20de\%20percepci\%C3\%B3n\%20sobre\%20 COVID-19\%20en\%20Paraguay.pdf

Unión Europea. (2006). Recomendación del Parlamento Europeoy del Consejo de 18 de diciembre de 2006 sobre las competencias clave para el aprendizaje permanente (2006/962/EC). Boletín Oficial. https://eur-lex.europa.eu/ legal-content/ES/TXT/PDF/?uri=CELEX:32006H0962\&from=EN

Villagrasa, C. (2009). Reflexiones en torno a la aplicación internacional de la Convención sobre los Derechos del Niño desde el derecho de participación: las pautas marcadas en los Congresos Mundiales sobre los Derechos de la Infancia y la Adolescencia. En C. Villagrasa, \& I. Ravetllat (Ed.), Por los derechos de la infancia y la adolescencia. Un compromiso desde la participación en el XX Aniversario de la Convención. Editorial Bosch.

\section{Sobre LOS AUTORES}

Claudia Sanabria Moudelle es Abogada por la Universidad Católica Nuestra Señora de la Asunción. Master en Derecho de Familia e Infancia, y en Globalización, Desarrollo y Cooperación, Universidad de Barcelona (España). Experta en Políticas Sociales de Infancia, Universidad Complutense de Madrid (España). Docente de grado y postgrado en la Universidad Iberoamericana. Evaluadora de revistas académicas. Coordinadora de la Maestría en Derecho de Familia - UNIBE. Encargada de Género, Ministerio de Salud Pública y Bienestar Social.

Eduardo Velázquez Romero es Abogado por la Universidad Nacional de Asunción. Máster en Estudios Internacionales: Organizaciones y Cooperación Internacional, Universidad de Barcelona (España). Máster en Derecho Civil y Comercial, Universidad Nacional de Asunción. Especialista en Didáctica Universitaria, Universidad Iberoamericana. Coordinador de Proyectos de Cooperación Internacional en Educación por la UNIBE. Director Académico de la Facultad de Postgrados de la Universidad Iberoamericana. 
Rev. cient. estud. investig. 9(1), 189-214; noviembre 2020

\section{Como cITAR}

Sanabria Moudelle, C., \& Velázquez Romero, E. (2020). Medidas educativas dispuestas en Paraguay en tiempos de pandemia desde la perspectiva de los derechos de la niñez y la adolescencia. Rev. cient. estud. investig., 9(1), 189-214. https://doi.org/10.26885/rcei.9.1.189 